\title{
Dimensionamento amostral para a estimação da média de precipitação pluvial mensal em locais do Estado do Mato Grosso do Sul
}

\author{
Sample size for estimating the means monthly rainfall in \\ locations of Mato Grosso do Sul State, Brazil
}

\author{
Francisco Eduardo Torres $^{\mathrm{I}}$ Alberto Cargnelutti FilhoII Paulo Eduardo Teodoro ${ }^{\mathrm{I}}$ \\ Caio Cezar Guedes Corrêa ${ }^{I}$ Larissa Pereira Ribeiro ${ }^{I}$ Elias Rodrigues da Cunha ${ }^{I I I}$
}

RESUMO

$O$ objetivo deste trabalho foi determinar o tamanho de amostra, em número de anos, para a estimação da média de precipitação pluvial mensal em locais do Estado de Mato Grosso do Sul e verificar sua variabilidade espaçotemporal. Utilizaram-se os dados de precipitação pluvial do periodo de 1954 a 2013, coletados do Sistema de Informações Hidrológicas da Agência Nacional de Águas - ANA. Em cada uma das 384 séries temporais (12 meses $\times 32$ locais), calcularam-se a média e o desvio padrão e testaram-se a aleatoriedade e a normalidade dos dados. Verificou-se a homogeneidade de variâncias entre os meses em cada local e entre os locais em cada mês. Calculou-se o tamanho de amostra em cada mês e local. O tamanho de amostra (número de anos) para a estimação da média de precipitação pluvial mensal é dependente do mês e do local. Para os meses e locais estudados, 63 anos de observações são suficientes para estimar a média de precipitação pluvial mensal, para um erro de estimação igual a 45\% da média estimada, com grau de confiança de $95 \%$.

Palavras-chave: série temporal, erro de estimação, amostragem.

\section{ABSTRACT}

The aim of this study was to determine the sample size, in number of years, to estimate the means monthly rainfall in locations of Mato Grosso do Sul and verify its space- temporal variability. It was used the rainfall data for the period 1954-2013, collected from the Hydrological Information System of the National Water Agency - ANA. The means and standard deviation were calculated for each of the 384 time series (12 months $\times 32$ locations) and the aleatory and normality data were tested. Then it was verified the homogeneity of variance among months in each locality and among locality in each month and it was determined the sample size to estimate the means monthly rainfall in each month and locality. The sample size (number of years) to estimate the means monthly rainfall is dependent on the month and locality. One concluded that 63 years of data are enough to predict the average monthly rainfall, with an estimation error equal to $45 \%$ of estimated average, with a degree confidence of $95 \%$

Key words: time series, error of estimation, sampling.

\section{INTRODUÇÃO}

O Estado de Mato Grosso do Sul tem na agropecuária a sua principal atividade econômica, com destaque para a produção de soja e bovinocultura, que se distribuem de forma distinta em dois biomas de atributos climáticos peculiares: Pantanal e Cerrado (CORREA et al.,

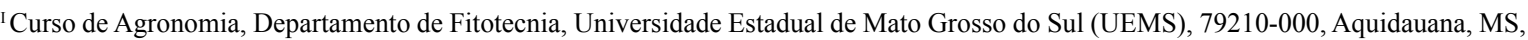
Brasil. E-mail: eduteodoro@hotmail.com. *Autor para correspondência.

IIDepartamento de Fitotecnia, Centro de Ciências Rurais (CCR), Universidade Federal de Santa Maria (UFSM), Santa Maria, RS, Brasil.

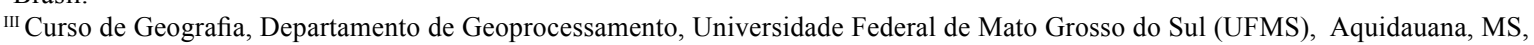
Brasil.
} 
2014). MINUZZI \& LOPEZ (2014) apontam a precipitação como uma das variáveis climáticas de maior influência no meio ambiente, na economia e na sociedade. Nesse contexto, é importante estimar sua disponibilidade, com a máxima precisão possível, em um determinado local e período do ano, para que, no planejamento de atividades, os riscos climáticos sejam minimizados.

De acordo com MARTIN et al. (2008), por meio da série histórica dos atributos do clima de uma região, é possível predizer as características do local, pois medidas de tendência central (média, mediana e moda), medidas de variabilidade dos dados (amplitude, desvio padrão, variância e coeficiente de variação) e medidas de assimetria e de curtose, descrevem o comportamento passado e podem ser usadas para previsões do clima.

Para estudos de variabilidade e de mudança climática, a World Meteorological Organization (WMO) preconiza que o número mínimo de anos de dados climáticos para a análise é de 30 anos, além de recomendar a utilização do Run Test (para indicar a aleatoriedade) (THOM, 1966). Entretanto, diversas pesquisas demonstram que o número mínimo de anos para determinação da média de um elemento meteorológico é estimado em função da variabilidade dos dados de cada local e da época do ano (CAMARGO \& HUBBARD, 1994; VERNICH \& ZUANNI, 1996; MARTIN et al., 2008; CARGNELUTTI FILHO et al., 2009b). Isso ocorre porque a estimativa obtida a partir de uma amostra está associada a um erro em razão da variabilidade das observações. Assim, um conjunto de dados com pouca variabilidade (homogêneo) necessitará de um menor tamanho de amostra para representá-los, quando comparado a um conjunto de dados heterogêneos (CARGNELUTTI FILHO et al., 2006).

Estudos sobre o dimensionamento amostral para estimativa da precipitação mensal são escassos no Brasil. Assim, o objetivo do trabalho foi determinar o tamanho de amostra, em número de anos, para a estimação da média de precipitação pluvial mensal, em locais do Estado de Mato Grosso do Sul, e verificar sua variabilidade espaço-temporal.

\section{MATERIAL E MÉTODOS}

Os dados de precipitação pluvial de 32 estações (locais) do Estado do Mato Grosso do Sul foram obtidos do Banco de Dados da Agência Nacional de Águas - ANA, coletados do período de 1954 a 2013 (Tabela 1). Em cada local e ano, somaram-se os dados diários de precipitação pluvial, obtendo-se a precipitação pluvial mensal, em mm mês ${ }^{-1}$, de cada um dos 12 meses do ano. Assim, formaram-se 384 séries temporais (12 meses $\times 32$ locais), com número diferenciado de anos de observações em cada série, definidas em função da disponibilidade de dados meteorológicos.

Em cada uma das 384 séries temporais de precipitação pluvial mensal, foram calculadas a média $(\bar{X})$ e o desvio padrão (s). A fim de identificar possíveis tendências de acréscimo ou decréscimo da precipitação pluvial mensal no período estudado, verificou-se a aleatoriedade dos dados em cada série temporal, por meio do teste de sequência (Run Test) (THOM, 1966; SIEGEL \& CASTELLAN JÚNIOR, 2006).

Foi aplicado um teste bilateral à hipótese $\mathrm{H}_{0}$ : a série é aleatória (sem tendência) versus a hipótese $\mathrm{H}_{1}$ : a série não é aleatória (com tendência). No teste, os dados de precipitação pluvial mensal foram utilizados em ordem cronológica, e o número de sequências foi calculado com base em valores menores e maiores que a mediana. A seguir, foi verificada a normalidade dos dados de cada série temporal, por meio do teste de Kolmogorov-Smirnov (SIEGEL \& CASTELLAN JÚNIOR, 2006).

Aplicou-se o teste de Bartlett (STEEL et al., 1997) aos dados de precipitação pluvial mensal, para verificar a homogeneidade de variâncias entre os meses do ano (12 variâncias - variabilidade temporal), em cada local (32 testes), e entre os locais (32 variâncias variabilidade espacial), em cada um dos 12 meses do ano (12 testes). Para cada série temporal de precipitação pluvial mensal, foi calculado o tamanho de amostra (n) para as semiamplitudes do intervalo de confiança (erros de estimação), fixadas em $25 \%, 35 \%$ e $45 \%$ da média $(\bar{x})$ de precipitação pluvial mensal, em $\mathrm{mm} \mathrm{mes}^{-1}$, ou seja, $0,25 \overline{\mathrm{X}}$ (maior precisão), 0,35 $\overline{\mathrm{X}}$ (precisão moderada) e $0,45 \overline{\mathrm{X}}$ (menor precisão), com grau de confiança $(1-\alpha)$ de $95 \%$, por meio da expressão $\mathrm{n}=\left[\left(\mathrm{t}_{\alpha / 2} \mathrm{~s}\right) /(\text { erro de estimação })\right]^{2}$ (BUSSAB \& MORETTIN, 2011), na qual $t_{\alpha / 2}$ é o valor crítico da distribuição t de Student, tal que $\mathrm{P}\left(\mathrm{t}>\mathrm{t}_{\alpha / 2}\right)=\alpha / 2$, com $\alpha=5 \%$ de probabilidade de erro e (n-1) graus de liberdade, sabendo que $\mathrm{s}$ é a estimativa do desvio-padrão. O tamanho de 
Tabela 1 - Altitude (em metros), latitude e longitude, período de observação da precipitação pluvial mensal e valor calculado da estatística do teste de Bartlett $\left(\chi_{\text {calc }}^{2}\right)$ das variâncias entre os meses, em cada local, e das variâncias entre os locais, em cada mês.

\begin{tabular}{|c|c|c|c|c|c|c|c|}
\hline Local & Altitude (m) & Latitude (S) & Longitude (W) & Período & $\chi_{\text {calc }}^{2}$ & Mês & $\chi_{\text {calc }}^{2}$ \\
\hline Água Clara & 376 & $-20^{\circ} 6^{\prime} 7^{\prime \prime}$ & $-52^{\circ} 55^{\prime} 33^{\prime \prime}$ & $1975-2013$ & $169,0^{*}$ & JAN & $40,9^{\text {ns }}$ \\
\hline Amambai & 395 & $-22^{\circ} 55^{\prime} 59^{\prime \prime}$ & $-55^{\circ} 13^{\prime} 0^{\prime \prime}$ & $1973-2013$ & $37,1 *$ & FEV & $60,4 *$ \\
\hline Anastácio & 106 & $-19^{\circ} 34^{\prime} 0^{\prime \prime}$ & $-56^{\circ} 12^{\prime} 0^{\prime \prime}$ & $1960-2000$ & $40,6^{*}$ & MAR & $59,5^{*}$ \\
\hline Anaurilândia & 284 & $-22^{\circ} 11^{\prime} 11^{\prime \prime}$ & $-52^{\circ} 42^{\prime} 48^{\prime \prime}$ & $1975-2013$ & $51,6^{*}$ & $\mathrm{ABR}$ & $118,2^{*}$ \\
\hline Aparecida do Taboado & 375 & $-20^{\circ} 16^{\prime}$ & $-51^{\circ} 6^{\prime} 13^{\prime \prime}$ & $1983-2013$ & $161,8^{*}$ & MAI & $242,7^{*}$ \\
\hline Aquidauana & 155 & $-20^{\circ} 27^{\prime} 24^{\prime \prime}$ & $-55^{\circ} 40^{\prime} 17^{\prime}$ & $1960-2000$ & $91,7 *$ & JUN & $265,4^{*}$ \\
\hline Bataguassu & 293 & $-21^{\circ} 43^{\prime} 33^{\prime \prime}$ & $-52^{\circ} 20^{\prime} 3^{\prime \prime}$ & $1975-2013$ & $58,8^{*}$ & JUL & $230,0^{*}$ \\
\hline Bodoquena & 133 & $-19^{\circ} 52^{\prime} 15^{\prime \prime}$ & $-56^{\circ} 59^{\prime} 1^{\prime \prime}$ & $1954-2013$ & $290,6^{*}$ & AGO & $280,0^{*}$ \\
\hline Caarapó & 454 & $-22^{\circ} 37^{\prime} 28^{\prime \prime}$ & $-54^{\circ} 49^{\prime} 29^{\prime \prime}$ & $1973-2013$ & $48,0^{*}$ & SET & $135,4^{*}$ \\
\hline Camapuã & 404 & $-19^{\circ} 29^{\prime} 48^{\prime \prime}$ & $-53^{\circ} 59^{\prime} 48^{\prime \prime}$ & $1973-2013$ & $115,0^{*}$ & OUT & $123,7^{*}$ \\
\hline Campo Grande & 559 & $-20^{\circ} 28^{\prime} 0^{\prime \prime}$ & $-54^{\circ} 40^{\prime} 0^{\prime \prime}$ & $1975-2013$ & $82,5^{*}$ & NOV & $74,3^{*}$ \\
\hline Chapadão do Sul & 570 & $-18^{\circ} 59^{\prime} 52^{\prime \prime}$ & $-52^{\circ} 35^{\prime} 17^{\prime \prime}$ & $1983-2013$ & $131,2 *$ & $\mathrm{DEZ}$ & $75,8 *$ \\
\hline Corumbá & 101 & $-17^{\circ} 37^{\prime} 24^{\prime \prime}$ & $-56^{\circ} 57^{\prime} 54^{\prime \prime}$ & $1983-2013$ & $95,8^{*}$ & & \\
\hline Costa Rica & 635 & $-18^{\circ} 32^{\prime} 50^{\prime \prime}$ & $-53^{\circ} 8^{\prime} 7^{\prime}$ & $1983-2013$ & $85,5^{*}$ & & \\
\hline Coxim & 250 & $-18^{\circ} 38^{\prime} 57^{\prime}$, & $-54^{\circ} 21^{\prime} 26^{\prime \prime}$ & $1973-2013$ & $171,5^{*}$ & & \\
\hline Dourados & 293 & $-22^{\circ} 23^{\prime} 53^{\prime \prime}$ & $-54^{\circ} 47^{\prime} 30^{\prime \prime}$ & $1973-2013$ & $86,8^{*}$ & & \\
\hline Glória de Dourados & 422 & $-22^{\circ} 24^{\prime} 21^{\prime \prime}$ & $-54^{\circ} 14^{\prime} 7^{\prime \prime}$ & $1973-2013$ & $25,5^{*}$ & & \\
\hline Iguatemi & 333 & $-23^{\circ} 40^{\prime} 55^{\prime \prime}$ & $-54^{\circ} 33^{\prime} 42^{\prime \prime}$ & $1975-2013$ & $49,3 *$ & & \\
\hline Inocência & 502 & $-19^{\circ} 44^{\prime} 11^{\prime \prime}$ & $-51^{\circ} 56^{\prime} 1^{\prime \prime}$ & $1983-2013$ & $119,5^{*}$ & & \\
\hline Maracaju & 356 & $-21^{\circ} 37^{\prime} 7^{\prime \prime}$ & $-55^{\circ} 8^{\prime} 13^{\prime \prime}$ & $1973-2013$ & $52,6^{*}$ & & \\
\hline Miranda & 140 & $-20^{\circ} 6^{\prime} 7^{\prime}$ & $-56^{\circ} 47^{\prime} 43^{\prime \prime}$ & $1960-2000$ & $236,3 *$ & & \\
\hline Navirai & 366 & $-23^{\circ} 3^{\prime} 28^{\prime \prime}$ & $-54^{\circ} 11^{\prime} 38^{\prime \prime}$ & $1975-2013$ & $36,6^{*}$ & & \\
\hline Nova Andradina & 271 & $-21^{\circ} 36^{\prime} 55^{\prime \prime}$ & $-53^{\circ} 3^{\prime} 8^{\prime \prime}$ & $1975-2013$ & $49,3 *$ & & \\
\hline Paranaíba & 458 & $-19^{\circ} 23^{\prime} 27^{\prime \prime}$ & $-51^{\circ} 36^{\prime} 32^{\prime \prime}$ & $1983-2013$ & $168,6^{*}$ & & \\
\hline Ponta Porã & 650 & $-22^{\circ} 32^{\prime} 0^{\prime \prime}$ & $-55^{\circ} 43^{\prime} 0^{\prime \prime}$ & $1973-2013$ & $79,1 *$ & & \\
\hline Porto Murtinho & 83 & $-21^{\circ} 42^{\prime} 5^{\prime}$ & $-57^{\circ} 53^{\prime} 30^{\prime \prime}$ & $1983-2013$ & $54,5^{*}$ & & \\
\hline Ribas do Rio Pardo & 373 & $-20^{\circ} 26^{\prime} 41^{\prime \prime}$ & $-53^{\circ} 45^{\prime} 29^{\prime \prime}$ & $1975-2013$ & $62,9 *$ & & \\
\hline Rio Brilhante & 287 & $-21^{\circ} 38^{\prime} 50^{\prime \prime}$ & $-54^{\circ} 25^{\prime} 31^{\prime \prime}$ & $1973-2013$ & $70,3^{*}$ & & \\
\hline Rio Negro & 233 & $-19^{\circ} 26^{\prime} 23^{\prime \prime}$ & $-54^{\circ} 59^{\prime} 0^{\prime \prime}$ & $1975-2013$ & $109,5^{*}$ & & \\
\hline Santa Rita do Pardo & 393 & $-21^{\circ} 17^{\prime} 43^{\prime \prime}$ & $-52^{\circ} 48^{\prime} 38^{\prime \prime}$ & $1975-2013$ & $47,6^{*}$ & & \\
\hline Selviria & 348 & $-20^{\circ} 21^{\prime} 49^{\prime \prime}$ & $-51^{\circ} 25^{\prime} 26^{\prime \prime}$ & $1983-2013$ & $107,5^{*}$ & & \\
\hline Três Lagoas & 313 & $-20^{\circ} 47^{\prime} 41^{\prime \prime}$ & $-51^{\circ} 42^{\prime} 46^{\prime \prime}$ & $1975-2013$ & 106,0 * & & \\
\hline
\end{tabular}

*Significativo a $5 \%$ de probabilidade de erro pelo teste de Bartlett. ${ }^{\text {ns }}=$ Não-significativo.

amostra foi calculado iterativamente até a sua convergência.

Em seguida, fixando-se $n$ igual ao número de anos de observações utilizadas no estudo, foi calculado o erro de estimação (semiamplitude do intervalo de confiança), em percentagem da estimativa da média $(\overline{\mathrm{X}})$ de precipitação pluvial mensal, para cada mês e local, por meio da expressão erro de estimação $=100[(\mathrm{t} \alpha / 2 \mathrm{~s}) /(\sqrt{\mathrm{n}} \overline{\mathrm{x}})$. As análises estatísticas foram realizadas com os aplicativos GENES (CRUZ, 2013) e Microsoft Office Excel ${ }^{\circledR}$.

\section{RESULTADOS E DISCUSSÃO}

Por meio do teste de Bartlett (STEEL et al., 1997), aplicado entre as variâncias dos 12 meses em cada local, constatou-se que estas foram heterogêneas nos 32 locais, o que indica tamanho de amostra diferenciado entre os 12 meses (Tabela 1). Entre as variâncias dos 32 locais, em cada mês, o teste de Bartlett revelou variância homogênea apenas para o mês de janeiro, o que pressupõe que o tamanho de amostra para estimar a precipitação média 
mensal, neste mês, pode ser o mesmo para os 32 locais. No entanto, para os demais 11 meses, as variâncias foram heterogêneas e, com isso, o tamanho de amostra, para mesma precisão, difere entre os locais. Assim, diante desses resultados, pode-se inferir que há variabilidade espacial e temporal do tamanho de amostra para determinação da precipitação média mensal.

A média da precipitação pluvial mensal, em mm mês ${ }^{-1}$, variou entre $11 \mathrm{~mm}$ (mês de julho em Aparecida do Taboado) e $305 \mathrm{~mm}$ (mês de janeiro em Inocência), sendo a média das 384 séries de $116 \mathrm{~mm}^{\text {mês }}{ }^{-1}$ (Tabela 2). As maiores e menores precipitações verificadas, que foram em janeiro e julho, respectivamente, são justificadas pelo clima do Estado, que, segundo a classificação de Köppen-Geiger, é do tipo Aw (tropical subúmido), caracterizado por chuvas no verão e períodos secos durante o inverno (CORRÊA et al., 2014). De maneira geral, nos 32 locais, maiores médias de precipitação pluvial mensal ocorreram nos primeiros e nos últimos meses do ano, com decréscimos gradativos em direção aos meses centrais do ano (Tabela 2).

Tabela 2 - Média da precipitação pluvial mensal, em mm mês ${ }^{-1}$, em locais do Estado do Mato Grosso do Sul, com dados meteorológicos disponíveis até 2013 .

\begin{tabular}{|c|c|c|c|c|c|c|c|c|c|c|c|c|}
\hline \multirow{2}{*}{ Local } & \multicolumn{12}{|c|}{ Mês } \\
\hline & JAN & FEV & MAR & $\mathrm{ABR}$ & MAI & JUN & JUL & AGO & SET & OUT & NOV & DEZ \\
\hline Água Clara & 273 & 230 & 186 & 77 & 70 & 24 & 22 & 30 & 68 & 124 & 178 & 239 \\
\hline Amambai & 164 & 163 & 149 & 144 & 136 & 110 & 51 & 66 & 116 & 206 & 191 & 202 \\
\hline Anastácio & 194 & 144 & 126 & 77 & 72 & 53 & 33 & 41 & 70 & 126 & 125 & 162 \\
\hline Anaurilândia & 185 & 172 & 139 & 99 & 89 & 62 & 41 & 44 & 114 & 128 & 128 & 163 \\
\hline Aparecida do Taboado & 238 & 169 & 155 & 74 & 55 & 24 & 11 & 19 & 58 & 92 & 112 & 188 \\
\hline Aquidauana & 178 & 153 & 123 & 101 & 97 & 46 & 20 & 29 & 85 & 108 & 162 & 175 \\
\hline Bataguassu & 203 & 171 & 131 & 90 & 86 & 53 & 36 & 32 & 84 & 106 & 135 & 186 \\
\hline Bodoquena & 199 & 137 & 131 & 79 & 56 & 37 & 21 & 19 & 48 & 93 & 130 & 165 \\
\hline Caarapó & 158 & 147 & 133 & 110 & 98 & 77 & 35 & 51 & 100 & 154 & 164 & 147 \\
\hline Camapuã & 258 & 193 & 189 & 84 & 85 & 32 & 22 & 27 & 83 & 129 & 165 & 216 \\
\hline Campo Grande & 208 & 181 & 147 & 87 & 91 & 43 & 33 & 40 & 84 & 134 & 158 & 209 \\
\hline Chapadão do Sul & 273 & 240 & 229 & 119 & 63 & 23 & 14 & 29 & 75 & 145 & 209 & 242 \\
\hline Corumbá & 181 & 157 & 143 & 70 & 43 & 30 & 16 & 18 & 42 & 69 & 148 & 170 \\
\hline Costa Rica & 300 & 229 & 245 & 142 & 68 & 20 & 14 & 22 & 67 & 149 & 195 & 242 \\
\hline Coxim & 244 & 208 & 148 & 77 & 55 & 26 & 16 & 17 & 56 & 122 & 171 & 216 \\
\hline Dourados & 185 & 174 & 141 & 85 & 90 & 78 & 38 & 48 & 103 & 138 & 174 & 167 \\
\hline Glória de Dourados & 181 & 166 & 156 & 117 & 109 & 87 & 49 & 60 & 124 & 155 & 154 & 158 \\
\hline Iguatemi & 166 & 147 & 119 & 120 & 132 & 93 & 62 & 68 & 119 & 173 & 163 & 181 \\
\hline Inocência & 305 & 258 & 206 & 97 & 57 & 23 & 14 & 30 & 71 & 103 & 152 & 231 \\
\hline Maracaju & 158 & 144 & 132 & 83 & 90 & 71 & 38 & 30 & 72 & 124 & 150 & 176 \\
\hline Miranda & 213 & 134 & 143 & 72 & 54 & 23 & 13 & 15 & 52 & 98 & 126 & 163 \\
\hline Navirai & 151 & 155 & 127 & 114 & 119 & 83 & 46 & 56 & 114 & 175 & 163 & 167 \\
\hline Nova Andradina & 218 & 203 & 163 & 97 & 104 & 61 & 41 & 45 & 92 & 134 & 162 & 192 \\
\hline Paranaíba & 281 & 201 & 208 & 90 & 52 & 24 & 14 & 17 & 52 & 113 & 138 & 238 \\
\hline Ponta Porã & 206 & 180 & 131 & 139 & 133 & 82 & 43 & 48 & 107 & 164 & 201 & 183 \\
\hline Porto Murtinho & 192 & 160 & 139 & 144 & 93 & 45 & 33 & 33 & 74 & 129 & 162 & 187 \\
\hline Ribas do Rio Pardo & 228 & 171 & 155 & 95 & 83 & 38 & 30 & 31 & 82 & 141 & 155 & 197 \\
\hline Rio Brilhante & 190 & 157 & 148 & 88 & 113 & 64 & 40 & 39 & 97 & 128 & 148 & 174 \\
\hline Rio Negro & 208 & 169 & 130 & 81 & 82 & 45 & 19 & 23 & 74 & 128 & 138 & 228 \\
\hline Santa Rita do Pardo & 217 & 163 & 142 & 86 & 86 & 53 & 36 & 34 & 86 & 124 & 141 & 180 \\
\hline Selviria & 238 & 198 & 162 & 78 & 54 & 30 & 14 & 23 & 64 & 96 & 124 & 176 \\
\hline Três Lagoas & 225 & 164 & 153 & 96 & 75 & 36 & 29 & 31 & 80 & 128 & 152 & 189 \\
\hline
\end{tabular}

Ciência Rural, v.46, n.1, jan, 2016. 
O desvio padrão da precipitação pluvial mensal oscilou entre $12 \mathrm{~mm}$ (mês de julho em Miranda) e $120 \mathrm{~mm}$ (mês de dezembro em Água Clara) e a média dos 384 desvios foi de $62 \mathrm{~mm}$ mês $^{-1}$ (Tabela 3). Resultados em magnitude superior $\left(77 \mathrm{~mm}\right.$ mês $^{-1}$ ) foram observados por CARGNELUTTI FILHO et al. (2009a) em pesquisa acerca do dimensionamento amostral para a estimação da média de precipitação mensal, em locais do Estado do Rio Grande do Sul. Nos 32 locais, de maneira geral, menores escores de desvio padrão ocorreram nos meses centrais do ano, com acréscimo gradativo em direção aos meses iniciais e finais do ano (Tabela 3 ).
Portanto, tanto as médias como o desvio padrão da precipitação pluvial mensal foram de elevada magnitude e, no mês de janeiro, diminuíram gradativamente até os meses de junho, julho e agosto e apresentaram aumento gradativo até o mês de dezembro (Tabelas 2 e 3 ).

De acordo com o teste de aleatoriedade (Run Test), a distribuição de 381 séries temporais de precipitação mensal $(99,22 \%)$ foi aleatória $(\mathrm{P}>0,01)$. Isso indica que, durante o período estudado, não houve tendência de acréscimo ou decréscimo da precipitação mensal nos 32 locais do Estado do Mato Grosso do Sul. O teste de normalidade (Kolmogorov-Smirnov)

Tabela 3 - Desvio padrão da precipitação pluvial mensal, em mm mês ${ }^{-1}$, em locais do Estado do Mato Grosso do Sul, com dados meteorológicos disponíveis até 2013.

\begin{tabular}{|c|c|c|c|c|c|c|c|c|c|c|c|c|}
\hline \multirow{2}{*}{ Local } & ------- & \multirow[b]{2}{*}{ FEV } & \multirow[b]{2}{*}{ MAR } & \multirow[b]{2}{*}{$\mathrm{ABR}$} & \multirow[b]{2}{*}{ MAI } & \multirow[b]{2}{*}{ JUN } & \multirow[b]{2}{*}{ JUL } & \multirow{2}{*}{ AGO } & \multirow[b]{2}{*}{ SET } & \multirow[b]{2}{*}{ OUT } & \multirow[b]{2}{*}{ NOV } & \multirow[b]{2}{*}{ DEZ } \\
\hline & JAN & & & & & & & & & & & \\
\hline Água Clara & 101 & 86 & 99 & 50 & 45 & 29 & 31 & 45 & 47 & 59 & 67 & 120 \\
\hline Amambai & 78 & 72 & 71 & 80 & 92 & 81 & 40 & 62 & 70 & 99 & 76 & 83 \\
\hline Anastácio & 68 & 57 & 66 & 48 & 43 & 48 & 32 & 41 & 49 & 63 & 53 & 64 \\
\hline Anaurilândia & 101 & 93 & 76 & 55 & 65 & 69 & 40 & 52 & 79 & 64 & 65 & 58 \\
\hline Aparecida do Taboado & 103 & 92 & 56 & 49 & 40 & 40 & 16 & 22 & 43 & 42 & 55 & 80 \\
\hline Aquidauana & 80 & 65 & 63 & 47 & 60 & 41 & 24 & 36 & 59 & 46 & 66 & 82 \\
\hline Bataguassu & 106 & 95 & 79 & 64 & 74 & 64 & 40 & 45 & 68 & 59 & 72 & 67 \\
\hline Bodoquena & 86 & 67 & 91 & 41 & 30 & 39 & 21 & 20 & 35 & 56 & 69 & 71 \\
\hline Caarapó & 69 & 62 & 78 & 67 & 68 & 70 & 28 & 47 & 65 & 71 & 74 & 65 \\
\hline Camapuã & 87 & 69 & 89 & 51 & 49 & 34 & 29 & 40 & 47 & 43 & 60 & 85 \\
\hline Campo Grande & 97 & 82 & 60 & 50 & 59 & 40 & 33 & 41 & 55 & 71 & 73 & 80 \\
\hline Chapadão do Sul & 78 & 95 & 99 & 58 & 42 & 38 & 19 & 42 & 56 & 46 & 92 & 98 \\
\hline Corumbá & 80 & 76 & 68 & 49 & 36 & 42 & 28 & 29 & 41 & 45 & 75 & 86 \\
\hline Costa Rica & 86 & 71 & 81 & 74 & 50 & 36 & 25 & 37 & 55 & 57 & 74 & 90 \\
\hline Coxim & 85 & 78 & 58 & 44 & 49 & 36 & 18 & 25 & 36 & 57 & 72 & 88 \\
\hline Dourados & 92 & 79 & 70 & 48 & 53 & 78 & 25 & 57 & 99 & 64 & 75 & 69 \\
\hline Glória de Dourados & 90 & 81 & 84 & 68 & 76 & 78 & 46 & 70 & 68 & 67 & 63 & 61 \\
\hline Iguatemi & 103 & 75 & 65 & 85 & 95 & 64 & 41 & 62 & 76 & 100 & 79 & 96 \\
\hline Inocência & 90 & 107 & 92 & 69 & 36 & 34 & 20 & 49 & 73 & 75 & 85 & 82 \\
\hline Maracaju & 75 & 72 & 77 & 54 & 73 & 66 & 35 & 38 & 56 & 64 & 72 & 81 \\
\hline Miranda & 76 & 55 & 56 & 31 & 33 & 17 & 12 & 14 & 36 & 56 & 49 & 61 \\
\hline Navirai & 72 & 79 & 76 & 72 & 100 & 76 & 41 & 55 & 73 & 87 & 84 & 71 \\
\hline Nova Andradina & 107 & 96 & 90 & 62 & 83 & 65 & 43 & 69 & 59 & 80 & 85 & 97 \\
\hline Paranaíba & 81 & 90 & 97 & 50 & 34 & 26 & 17 & 20 & 49 & 50 & 57 & 61 \\
\hline Ponta Porã & 80 & 76 & 62 & 60 & 78 & 56 & 27 & 34 & 62 & 60 & 42 & 58 \\
\hline Porto Murtinho & 84 & 70 & 76 & 80 & 80 & 46 & 31 & 48 & 63 & 82 & 62 & 95 \\
\hline Ribas do Rio Pardo & 87 & 60 & 73 & 55 & 56 & 49 & 32 & 37 & 67 & 60 & 71 & 77 \\
\hline Rio Brilhante & 90 & 66 & 77 & 42 & 59 & 50 & 32 & 43 & 61 & 48 & 45 & 58 \\
\hline Rio Negro & 82 & 72 & 75 & 52 & 72 & 50 & 20 & 29 & 59 & 47 & 53 & 77 \\
\hline Santa Rita do Pardo & 89 & 63 & 65 & 49 & 59 & 57 & 37 & 46 & 62 & 59 & 58 & 86 \\
\hline Selviria & 80 & 81 & 87 & 53 & 41 & 50 & 16 & 30 & 51 & 49 & 58 & 76 \\
\hline Três Lagoas & 115 & 81 & 72 & 74 & 60 & 34 & 31 & 42 & 59 & 64 & 64 & 82 \\
\hline
\end{tabular}


revelou que os dados de 359 séries temporais de precipitação mensal $(93,49 \%)$ se ajustaram à distribuição normal $(\mathrm{P}>0,01)$. Em face desses elevados percentuais de séries aleatórias e com ajuste à distribuição normal, pode-se inferir que os dados destas séries temporais de precipitação mensal tem credibilidade para o estudo do tamanho de amostra (SIEGEL \& CASTELLAN JÚNIOR, 2006).

O tamanho de amostra (número de anos), para a estimação da média de precipitação mensal, em cada mês e local, com semiamplitude do intervalo de confiança igual a $25 \%$ da média estimada, em $\mathrm{mm} \mathrm{mês}^{-1}$, e grau de confiança de $95 \%$, oscilou entre 200 (mês de junho em Costa Rica) e 3 anos (mês de novembro em Ponta Porã) (Tabela 4). Para a estimação da média de precipitação mensal, em cada mês e local, com semiamplitude do intervalo de confiança igual a $35 \%$ da média estimada, em mm mês ${ }^{-1}$, e grau de confiança de $95 \%$, o tamanho da amostra variou entre 103 (mês de junho em Costa Rica) e um ano (mês de novembro em Ponta Porã) (Tabela 5). Esses resultados reforçam a hipótese de variabilidade do tamanho de amostra entre meses dentro dos locais (temporal) e entre locais dentro de meses (espacial). Em termos práticos, existem dificuldades de obter séries históricas de atributos do clima com, no mínimo, 103 anos de observações para uma determinada região. Dessa forma, menor tamanho de amostra foi dimensionado com base em semiamplitudes do intervalo de confiança igual a 45\% (Tabela 6) da estimativa da média $(\overline{\mathrm{X}})$ de precipitação mensal. Assim, tomando-se como referência o

Tabela 4 - Tamanho de amostra (número de anos) para a estimação da média de precipitação pluvial mensal em locais do Estado do Mato Grosso do Sul, para a semiamplitude do intervalo de confiança (erro de estimação) igual $25 \%$ da média ( $\overline{\mathrm{X}}$ ), ou seja, $0,25 \overline{\mathrm{X}}$.

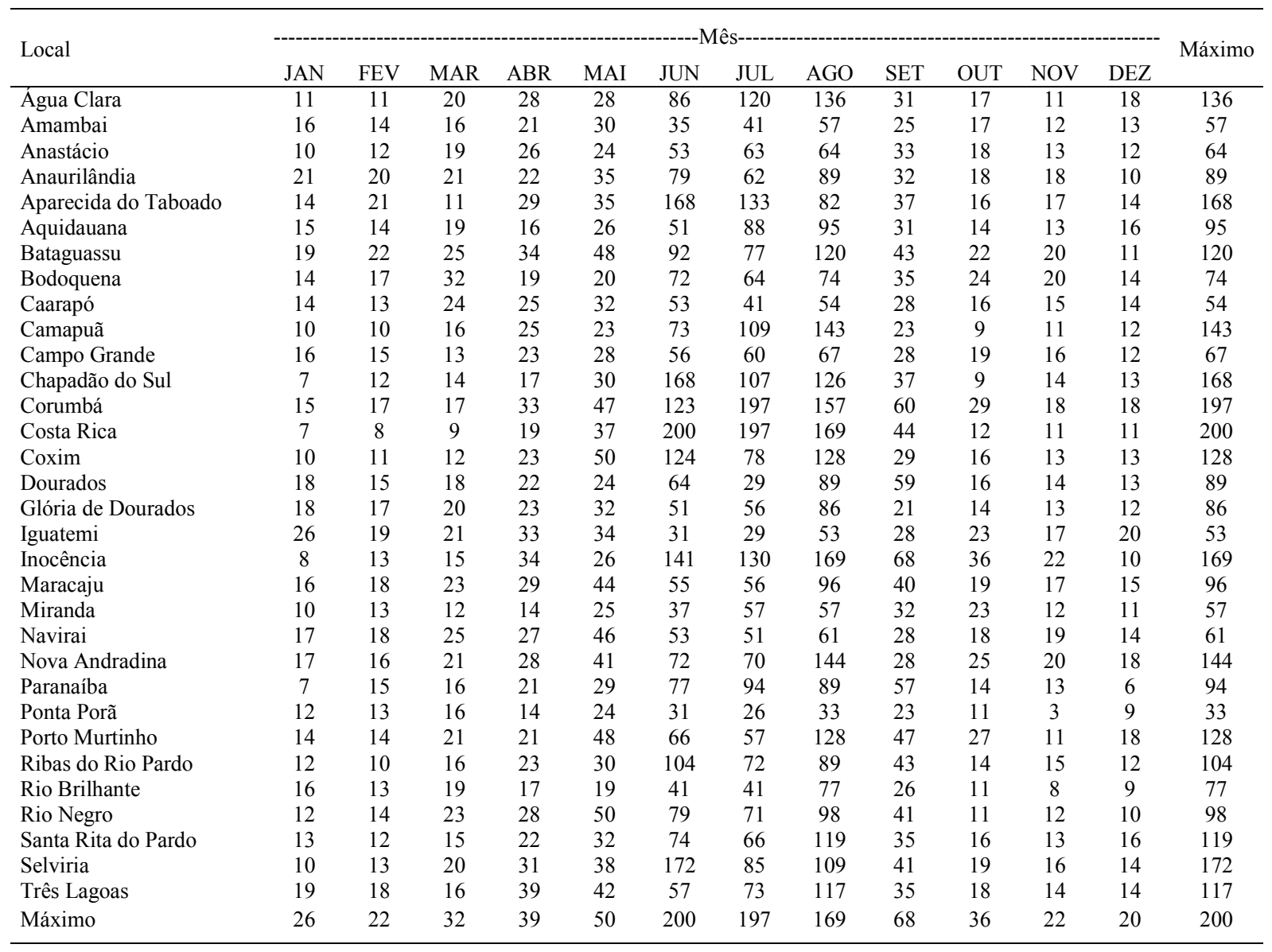


Tabela 5 - Tamanho de amostra (número de anos) para a estimação da média de precipitação pluvial mensal em locais do Estado do Mato Grosso do Sul, para a semiamplitude do intervalo de confiança (erro de estimação) igual 35\% da média ( $\overline{\mathrm{X}}$ ), ou seja, $0,35 \overline{\mathrm{X}}$.

\begin{tabular}{|c|c|c|c|c|c|c|c|c|c|c|c|c|c|}
\hline \multirow{2}{*}{ Local } & \multirow{2}{*}{ JAN } & \multirow[b]{2}{*}{ FEV } & \multirow[b]{2}{*}{ MAR } & \multirow[b]{2}{*}{$\mathrm{ABR}$} & \multirow[b]{2}{*}{ MAI } & \multirow[b]{2}{*}{ JUN } & \multirow[b]{2}{*}{ JUL } & \multirow[b]{2}{*}{ AGO } & \multirow[b]{2}{*}{ SET } & \multirow[b]{2}{*}{ OUT } & \multirow[b]{2}{*}{$\mathrm{NOV}$} & \multirow[b]{2}{*}{ DEZ } & \multirow{2}{*}{ Máximo } \\
\hline & & & & & & & & & & & & & \\
\hline Água Clara & 6 & 6 & 12 & 16 & 16 & 45 & 62 & 71 & 17 & 10 & 6 & 10 & 71 \\
\hline Amambai & 10 & 9 & 10 & 12 & 17 & 19 & 22 & 30 & 14 & 10 & 7 & 7 & 30 \\
\hline Anastácio & 6 & 7 & 11 & 15 & 13 & 28 & 33 & 34 & 18 & 10 & 8 & 7 & 34 \\
\hline Anaurilândia & 12 & 12 & 12 & 12 & 19 & 41 & 33 & 46 & 18 & 11 & 11 & 6 & 46 \\
\hline Aparecida do Taboado & 8 & 12 & 6 & 16 & 19 & 87 & 69 & 43 & 20 & 9 & 10 & 8 & 87 \\
\hline Aquidauana & 9 & 8 & 11 & 10 & 14 & 27 & 46 & 50 & 17 & 8 & 8 & 10 & 50 \\
\hline Bataguassu & 11 & 12 & 14 & 19 & 26 & 48 & 40 & 62 & 23 & 12 & 12 & 6 & 62 \\
\hline Bodoquena & 8 & 10 & 18 & 11 & 11 & 38 & 34 & 39 & 19 & 14 & 12 & 8 & 39 \\
\hline Caarapó & 8 & 8 & 13 & 14 & 18 & 28 & 22 & 29 & 16 & 9 & 9 & 9 & 29 \\
\hline Camapuã & 5 & 6 & 10 & 14 & 13 & 38 & 57 & 74 & 13 & 5 & 6 & 7 & 74 \\
\hline Campo Grande & 10 & 9 & 8 & 13 & 16 & 30 & 32 & 35 & 16 & 11 & 9 & 6 & 35 \\
\hline Chapadão do Sul & 3 & 7 & 8 & 10 & 16 & 87 & 56 & 66 & 20 & 4 & 9 & 7 & 87 \\
\hline Corumbá & 9 & 10 & 10 & 18 & 25 & 64 & 102 & 81 & 32 & 16 & 11 & 11 & 102 \\
\hline Costa Rica & 3 & 4 & 5 & 11 & 20 & 103 & 102 & 87 & 24 & 7 & 6 & 6 & 103 \\
\hline Coxim & 6 & 6 & 7 & 13 & 27 & 64 & 41 & 66 & 16 & 9 & 8 & 8 & 66 \\
\hline Dourados & 10 & 9 & 10 & 13 & 14 & 34 & 16 & 47 & 31 & 9 & 8 & 8 & 47 \\
\hline Glória de Dourados & 10 & 10 & 12 & 13 & 18 & 27 & 30 & 45 & 12 & 8 & 8 & 6 & 45 \\
\hline Iguatemi & 15 & 11 & 12 & 18 & 19 & 17 & 16 & 28 & 15 & 13 & 10 & 11 & 28 \\
\hline Inocência & 3 & 8 & 9 & 19 & 15 & 73 & 68 & 87 & 36 & 19 & 12 & 6 & 87 \\
\hline Maracaju & 10 & 10 & 13 & 16 & 23 & 29 & 30 & 50 & 22 & 11 & 10 & 9 & 50 \\
\hline Miranda & 6 & 8 & 7 & 8 & 14 & 20 & 30 & 30 & 18 & 13 & 7 & 6 & 30 \\
\hline Navirai & 10 & 11 & 14 & 15 & 25 & 28 & 27 & 32 & 16 & 10 & 11 & 8 & 32 \\
\hline Nova Andradina & 10 & 10 & 12 & 15 & 22 & 38 & 37 & 75 & 15 & 14 & 11 & 11 & 75 \\
\hline Paranaíba & 3 & 9 & 9 & 12 & 16 & 40 & 49 & 47 & 30 & 9 & 8 & 2 & 49 \\
\hline Ponta Porã & 6 & 8 & 10 & 8 & 13 & 17 & 14 & 18 & 13 & 6 & 1 & 4 & 18 \\
\hline Porto Murtinho & 9 & 8 & 12 & 12 & 26 & 35 & 30 & 67 & 25 & 15 & 6 & 11 & 67 \\
\hline Ribas do Rio Pardo & 6 & 6 & 10 & 13 & 16 & 54 & 38 & 47 & 23 & 8 & 9 & 7 & 54 \\
\hline Rio Brilhante & 10 & 8 & 11 & 10 & 11 & 22 & 22 & 40 & 15 & 6 & 4 & 5 & 40 \\
\hline Rio Negro & 7 & 8 & 13 & 15 & 27 & 41 & 38 & 51 & 22 & 6 & 6 & 5 & 51 \\
\hline Santa Rita do Pardo & 8 & 6 & 9 & 13 & 17 & 39 & 35 & 62 & 19 & 10 & 8 & 10 & 62 \\
\hline Selviria & 5 & 8 & 12 & 17 & 21 & 89 & 45 & 57 & 22 & 11 & 9 & 8 & 89 \\
\hline Três Lagoas & 11 & 10 & 10 & 21 & 23 & 31 & 38 & 61 & 19 & 10 & 8 & 8 & 61 \\
\hline Máximo & 15 & 12 & 18 & 21 & 27 & 103 & 102 & 87 & 36 & 19 & 12 & 11 & 103 \\
\hline
\end{tabular}

maior tamanho de amostra (mês de junho em Costa Rica e mês de julho em Corumbá e Costa Rica), pode-se inferir, com $95 \%$ de confiança, que, com o uso de 63 anos de observações, o erro máximo na estimativa da média $(\overline{\mathrm{X}})$ de precipitação pluvial mensal será de $\pm 45 \%$ de $\overline{\mathrm{X}}$, independentemente do mês e local.

Estes resultados corroboram os obtidos por CAMARGO \& HUBBARD (1994), MARTIN et al. (2008) e CARGNELUTTI FILHO et al. (2009b), que constataram variabilidade temporal e espacial do número de anos para estimativas da média de elementos meteorológicos para o estado de Minas Gerais, São Paulo e Rio Grande do Sul, respectivamente. Com isso, o uso de séries temporais de 30 anos, de forma generalizada, para estimar a média de um elemento meteorológico, pode não contemplar a variabilidade dos dados de cada local e em cada época do ano, o que pode gerar estimativas com precisões diferenciadas.

Com os anos de observações utilizados neste estudo, o erro de estimação 
Tabela 6 - Tamanho de amostra (número de anos) para a estimação da média de precipitação pluvial mensal em locais do Estado do Mato Grosso do Sul, para a semiamplitude do intervalo de confiança (erro de estimação) igual 45\% da média ( $\overline{\mathrm{X}}$ ), ou seja, $0,45 \overline{\mathrm{X}}$.

\begin{tabular}{|c|c|c|c|c|c|c|c|c|c|c|c|c|c|}
\hline \multirow{2}{*}{ Local } & \multicolumn{12}{|c|}{ 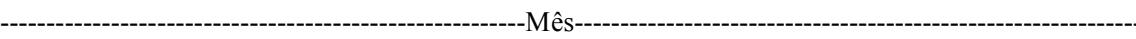 } & \multirow{2}{*}{ Máximo } \\
\hline & JAN & FEV & MAR & $\mathrm{ABR}$ & MAI & JUN & JUL & AGO & SET & OUT & NOV & DEZ & \\
\hline Água Clara & 3 & 3 & 8 & 11 & 11 & 28 & 39 & 44 & 11 & 6 & 3 & 7 & 44 \\
\hline Amambai & 6 & 5 & 6 & 8 & 11 & 13 & 14 & 19 & 10 & 6 & 4 & 4 & 19 \\
\hline Anastácio & 2 & 4 & 8 & 10 & 9 & 18 & 21 & 21 & 12 & 7 & 4 & 4 & 21 \\
\hline Anaurilândia & 8 & 8 & 8 & 8 & 13 & 26 & 21 & 29 & 12 & 7 & 7 & 2 & 29 \\
\hline Aparecida do Taboado & 5 & 8 & 2 & 11 & 13 & 54 & 43 & 27 & 13 & 6 & 6 & 5 & 54 \\
\hline Aquidauana & 6 & 5 & 7 & 6 & 10 & 17 & 29 & 31 & 12 & 5 & 4 & 6 & 31 \\
\hline Bataguassu & 8 & 8 & 10 & 12 & 17 & 30 & 25 & 39 & 15 & 8 & 8 & 3 & 39 \\
\hline Bodoquena & 5 & 6 & 12 & 7 & 8 & 24 & 21 & 25 & 13 & 9 & 8 & 5 & 25 \\
\hline Caarapó & 5 & 4 & 9 & 10 & 12 & 18 & 14 & 19 & 11 & 6 & 6 & 5 & 19 \\
\hline Camapuã & 2 & 2 & 6 & 9 & 9 & 24 & 35 & 46 & 9 & 2 & 3 & 4 & 46 \\
\hline Campo Grande & 6 & 6 & 4 & 9 & 11 & 19 & 20 & 22 & 11 & 8 & 6 & 3 & 22 \\
\hline Chapadão do Sul & 2 & 4 & 5 & 6 & 11 & 54 & 35 & 41 & 13 & 2 & 5 & 4 & 54 \\
\hline Corumbá & 6 & 6 & 6 & 12 & 16 & 40 & 63 & 50 & 20 & 11 & 7 & 7 & 63 \\
\hline Costa Rica & 2 & 2 & 2 & 8 & 13 & 63 & 63 & 54 & 15 & 3 & 3 & 3 & 63 \\
\hline Coxim & 2 & 3 & 4 & 9 & 17 & 40 & 26 & 41 & 11 & 6 & 4 & 4 & 41 \\
\hline Dourados & 7 & 6 & 7 & 9 & 9 & 21 & 11 & 29 & 20 & 6 & 5 & 4 & 29 \\
\hline Glória de Dourados & 7 & 6 & 8 & 9 & 12 & 18 & 19 & 28 & 8 & 5 & 4 & 3 & 28 \\
\hline Iguatemi & 10 & 7 & 8 & 12 & 12 & 11 & 11 & 18 & 10 & 9 & 6 & 8 & 18 \\
\hline Inocência & 2 & 4 & 6 & 12 & 10 & 45 & 42 & 54 & 23 & 13 & 8 & 2 & 54 \\
\hline Maracaju & 6 & 7 & 9 & 11 & 15 & 19 & 19 & 31 & 14 & 7 & 6 & 6 & 31 \\
\hline Miranda & 2 & 4 & 4 & 5 & 10 & 13 & 19 & 19 & 12 & 9 & 4 & 3 & 19 \\
\hline Navirai & 6 & 7 & 9 & 10 & 16 & 18 & 18 & 21 & 10 & 7 & 7 & 5 & 21 \\
\hline Nova Andradina & 6 & 6 & 8 & 10 & 15 & 24 & 23 & 46 & 10 & 9 & 7 & 7 & 46 \\
\hline Paranaíba & 2 & 6 & 6 & 8 & 11 & 25 & 31 & 29 & 19 & 5 & 4 & 1 & 31 \\
\hline Ponta Porã & 3 & 4 & 6 & 5 & 9 & 11 & 10 & 12 & 9 & 3 & 1 & 2 & 12 \\
\hline Porto Murtinho & 5 & 5 & 8 & 8 & 17 & 22 & 19 & 41 & 16 & 10 & 3 & 7 & 41 \\
\hline Ribas do Rio Pardo & 3 & 2 & 6 & 9 & 11 & 34 & 24 & 29 & 15 & 5 & 6 & 4 & 34 \\
\hline Rio Brilhante & 6 & 4 & 7 & 6 & 7 & 14 & 15 & 25 & 10 & 3 & 2 & 2 & 25 \\
\hline Rio Negro & 4 & 5 & 9 & 10 & 17 & 26 & 24 & 32 & 15 & 3 & 4 & 2 & 32 \\
\hline Santa Rita do Pardo & 4 & 3 & 6 & 9 & 12 & 25 & 22 & 38 & 13 & 6 & 4 & 6 & 38 \\
\hline Selviria & 2 & 4 & 8 & 12 & 13 & 55 & 28 & 35 & 15 & 7 & 6 & 5 & 55 \\
\hline Três Lagoas & 7 & 6 & 6 & 14 & 15 & 19 & 24 & 38 & 13 & 7 & 4 & 5 & 38 \\
\hline Máximo & 10 & 8 & 12 & 14 & 17 & 63 & 63 & 54 & 23 & 13 & 8 & 8 & 63 \\
\hline
\end{tabular}

(semiamplitude do intervalo de confiança), em percentagem da estimativa da média $(\overline{\mathrm{X}})$ de precipitação pluvial mensal, oscilou entre 65,8\% (mês de junho em Costa Rica) e 6,5\% (mês de novembro em Ponta Porã) (Tabela 7). De maneira geral, com as séries históricas dos locais utilizados neste trabalho, menor precisão é obtida nos meses centrais do ano (junho, julho e agosto), com aumento gradativo da precisão (diminuição do erro de estimação) em direção aos meses iniciais e finais do ano.

\section{CONCLUSÃO}

O tamanho de amostra, em número de anos, para a estimação da média de precipitação pluvial mensal, no Estado do Mato Grosso do Sul, é dependente do mês e do local. Para os meses e locais estudados, 63 anos de observações são suficientes para estimar a média de precipitação pluvial mensal, para um erro de estimação igual a $45 \%$ da média estimada, com grau de confiança de $95 \%$.

Ciência Rural, v.46, n.1, jan, 2016. 
Tabela 7 - Número de anos de observações utilizadas no estudo (n) e erro de estimação (semiamplitude do intervalo de confiança), em percentagem da estimativa da média $(\overline{\mathrm{X}})$ de precipitação pluvial mensal, em cada mês e local do Estado do Mato Grosso do Sul.

\begin{tabular}{|c|c|c|c|c|c|c|c|c|c|c|c|c|c|c|}
\hline \multirow{2}{*}{ Local } & \multirow{2}{*}{$\mathrm{n}$} & \multicolumn{12}{|l|}{$-\cdots$} & \multirow{2}{*}{ Média } \\
\hline & & JAN & FEV & MAR & $\mathrm{ABR}$ & MAI & JUN & JUL & AGO & SET & OUT & $\mathrm{NOV}$ & DEZ & \\
\hline Água Clara & 39 & 12,0 & 12,2 & 17,3 & 21,0 & 20,9 & 37,8 & 44,8 & 47,8 & 22,2 & 15,5 & 12,3 & 16,2 & 23,3 \\
\hline Amambai & 41 & 15,0 & 13,9 & 15,0 & 17,5 & 21,3 & 23,1 & 24,9 & 29,6 & 19,3 & 15,2 & 12,5 & 12,9 & 18,4 \\
\hline Anastácio & 41 & 11,1 & 12,6 & 16,6 & 19,6 & 18,6 & 28,5 & 31,3 & 31,6 & 22,3 & 15,7 & 13,3 & 12,5 & 19,5 \\
\hline Anaurilândia & 39 & 17,8 & 17,4 & 17,8 & 18,1 & 23,6 & 36,1 & 32,0 & 38,4 & 22,6 & 16,3 & 16,5 & 11,5 & 22,3 \\
\hline Aparecida do Taboado & 31 & 15,8 & 20,0 & 13,1 & 24,1 & 26,9 & 60,2 & 53,5 & 41,8 & 27,3 & 16,9 & 17,9 & 15,6 & 27,8 \\
\hline Aquidauana & 41 & 14,2 & 13,4 & 16,3 & 14,9 & 19,4 & 28,0 & 37,3 & 38,8 & 21,6 & 13,4 & 12,9 & 14,8 & 20,4 \\
\hline Bataguassu & 39 & 17,0 & 18,0 & 19,5 & 23,2 & 27,9 & 39,1 & 35,7 & 44,8 & 26,2 & 18,0 & 17,3 & 11,7 & 24,9 \\
\hline Bodoquena & 60 & 11,2 & 12,6 & 17,9 & 13,4 & 13,7 & 27,6 & 25,8 & 27,8 & 18,8 & 15,4 & 13,8 & 11,1 & 17,4 \\
\hline Caarapó & 41 & 13,8 & 13,3 & 18,5 & 19,3 & 21,8 & 28,6 & 25,0 & 29,0 & 20,5 & 14,5 & 14,3 & 13,8 & 19,4 \\
\hline Camapuã & 41 & 10,6 & 11,2 & 14,8 & 19,0 & 18,1 & 33,7 & 41,6 & 47,8 & 18,0 & 10,5 & 11,5 & 12,4 & 20,8 \\
\hline Campo Grande & 39 & 15,2 & 14,6 & 13,1 & 18,6 & 21,1 & 30,3 & 31,5 & 33,2 & 21,1 & 17,0 & 15,0 & 12,5 & 20,3 \\
\hline Chapadão do Sul & 31 & 10,5 & 14,5 & 15,9 & 18,0 & 24,4 & 60,3 & 47,8 & 52,1 & 27,5 & 11,7 & 16,1 & 14,8 & 26,1 \\
\hline Corumbá & 31 & 16,2 & 17,8 & 17,5 & 25,8 & 31,2 & 51,4 & 65,3 & 58,2 & 35,5 & 24,1 & 18,6 & 18,5 & 31,7 \\
\hline Costa Rica & 31 & 10,6 & 11,3 & 12,2 & 19,2 & 27,3 & 65,8 & 65,3 & 60,3 & 30,2 & 14,1 & 13,9 & 13,7 & 28,7 \\
\hline Coxim & 41 & 11,0 & 11,9 & 12,4 & 18,2 & 27,7 & 44,3 & 35,0 & 45,1 & 20,6 & 14,6 & 13,2 & 12,8 & 22,2 \\
\hline Dourados & 41 & 15,7 & 14,3 & 15,7 & 17,9 & 18,7 & 31,6 & 20,6 & 37,5 & 30,2 & 14,5 & 13,7 & 13,1 & 20,3 \\
\hline Glória de Dourados & 41 & 15,7 & 15,5 & 17,0 & 18,3 & 22,0 & 28,1 & 29,4 & 36,7 & 17,4 & 13,7 & 12,9 & 12,1 & 19,9 \\
\hline Iguatemi & 39 & 20,1 & 16,7 & 17,7 & 22,8 & 23,4 & 22,2 & 21,5 & 29,4 & 20,8 & 18,7 & 15,7 & 17,1 & 20,5 \\
\hline Inocência & 31 & 10,8 & 15,2 & 16,4 & 26,4 & 22,8 & 55,0 & 52,8 & 60,3 & 37,9 & 26,9 & 20,5 & 13,0 & 29,8 \\
\hline Maracaju & 41 & 15,0 & 15,8 & 18,4 & 20,6 & 25,8 & 29,3 & 29,5 & 38,9 & 24,6 & 16,4 & 15,1 & 14,5 & 22,0 \\
\hline Miranda & 41 & 11,2 & 12,9 & 12,4 & 13,5 & 19,3 & 23,5 & 29,7 & 29,7 & 21,9 & 18,1 & 12,2 & 11,8 & 18,0 \\
\hline Navirai & 39 & 15,5 & 16,5 & 19,5 & 20,6 & 27,3 & 29,4 & 28,8 & 31,6 & 20,9 & 16,2 & 16,7 & 13,9 & 21,4 \\
\hline Nova Andradina & 39 & 15,9 & 15,3 & 17,9 & 20,8 & 25,8 & 34,6 & 34,0 & 49,2 & 20,8 & 19,4 & 17,1 & 16,5 & 23,9 \\
\hline Paranaíba & 31 & 10,5 & 16,4 & 17,0 & 20,3 & 24,3 & 40,3 & 44,9 & 43,5 & 34,5 & 16,1 & 15,1 & 9,4 & 24,4 \\
\hline Ponta Porã & 41 & 12,2 & 13,3 & 14,9 & 13,7 & 18,5 & 21,5 & 19,4 & 22,2 & 18,3 & 11,5 & 6,5 & 10,0 & 15,2 \\
\hline Porto Murtinho & 31 & 16,1 & 16,0 & 20,0 & 20,3 & 31,6 & 37,4 & 34,4 & 52,5 & 31,2 & 23,2 & 13,9 & 18,6 & 26,3 \\
\hline Ribas do Rio Pardo & 39 & 12,4 & 11,5 & 15,3 & 18,6 & 21,6 & 41,6 & 34,4 & 38,5 & 26,4 & 13,9 & 14,8 & 12,7 & 21,8 \\
\hline Rio Brilhante & 41 & 15,0 & 13,2 & 16,4 & 15,1 & 16,6 & 24,9 & 25,1 & 34,7 & 19,7 & 11,8 & 9,7 & 10,5 & 17,7 \\
\hline Rio Negro & 39 & 12,8 & 13,7 & 18,8 & 20,7 & 28,5 & 36,1 & 34,3 & 40,5 & 25,8 & 11,9 & 12,5 & 10,9 & 22,2 \\
\hline Santa Rita do Pardo & 39 & 13,3 & 12,5 & 14,8 & 18,3 & 22,3 & 35,1 & 33,1 & 44,6 & 23,6 & 15,3 & 13,4 & 15,4 & 21,8 \\
\hline Selviria & 31 & 12,3 & 14,9 & 19,7 & 25,2 & 27,8 & 60,9 & 42,5 & 48,3 & 29,1 & 18,7 & 17,1 & 15,8 & 27,7 \\
\hline Três Lagoas & 39 & 16,6 & 16,0 & 15,3 & 25,0 & 25,9 & 30,6 & 34,7 & 44,2 & 23,6 & 16,2 & 13,7 & 14,0 & 23,0 \\
\hline Média & & 13,8 & 14,5 & 16,4 & 19,6 & 23,3 & 36,8 & 35,8 & 40,9 & 24,4 & 16,1 & 14,4 & 13,6 & 22,5 \\
\hline
\end{tabular}

\section{AGRADECIMENTOS}

À Agência Nacional de Águas (ANA), pelo fornecimento de dados para a presente pesquisa. Ao Conselho Nacional de Desenvolvimento Científico e Tecnológico (CNPq) e à Coordenação de Aperfeiçoamento de Pessoal de Nível Superior (CAPES), pelas bolsas concedidas.

\section{REFERÊNCIAS}

BUSSAB, W.O.; MORETTIN, P.A. Estatística básica. 7.ed. São Paulo: Saraiva, 2011. 540p.

CAMARGO, M.B.P.; HUBBARD, K.G. Spatial and temporal variability of daily weather variables in sub-humid and semi-arid areas of the united states high plains. Agricultural and Forest Meteorology, v.68, p.29-41, 1994. Disponível em: <http://dx.doi. org/10.1016/S0168-1923(98)00122-1>. Acesso em: 25 jun. 2014. doi: 10.1016/S0168-1923(98)00122-1.

CARGNELUTTI FILHO, A. et al. Variabilidade temporal e espacial do tamanho de amostra para estimativa das médias mensais de temperatura máxima do ar no Estado do Rio Grande do Sul. Revista Brasileira de Agrometeorologia, v.14, p.87-95, 2006.

CARGNELUTTI FILHO, A. et al. Dimensionamento da amostra para a estimação da média de precipitação pluvial mensal em diferentes locais do Estado do Rio Grande do Sul. Ciência Rural, v.40, p.12-19, 2009a. Disponível em: <http://www.scielo.br/ scielo.php?script $=$ sci_arttext\&pid $=$ S0103-84782010000100003 $>$. Acesso em:20jun. 2014. doi: 10.1590/S0103-84782009005000226. 
CARGNELUTTI FILHO, A. et al. Variabilidade temporal e espacial da precisão das estimativas de elementos meteorológicos no Rio Grande do Sul. Ciência Rural, v.39, p.962-970, 2009 b. Disponível em: <http://www.scielo.br/scielo.php?script=sci_artte xt\&pid=S0103-84782009000400002 > . Acesso em: 19 jun. 2014. doi: $10.1590 / \mathrm{S} 0103-84782009005000051$.

CORREA, C.C.G. et al. Spatial interpolation of annual rainfall in the State Mato Grosso do Sul (Brazil) using different transitive theoretical mathematical models. International Journal of Innovative Research in Science, Engineering and Technology, v.3, p.16618-16625, 2014. Disponível em: <http://www.rroij.com/ open-access/spatial-interpolation-of-annual-rainfall-in-thestatemato-grosso-do-sul-brazil-usingdifferent-transitive-theoreticalmathematicalmodels.pdf $>$. Acesso em: 15 jun. 2014 . doi: 10.15680/IJIRSET.2014.0310006

CRUZ, C.D. GENES - a software package for analysis in experimental statistics and quantitative genetics. Acta Scientiarum Agronomy, v.35, p.271-276, 2013. Disponível em: $<$ http://dx.doi org/10.4025/actasciagron.v35i3.21251. Acesso em: 22 mai. 2014. doi: 10.4025/actasciagron.v35i3.21251.

MARTIN, T.N. et al. Regiões homogêneas e tamanho de amostra para atributos do clima no Estado de São Paulo,
Brasil. Ciência Rural, v.38, p.690-697, 2008. Disponível em: $<$ http://www.scielo.br/scielo.php?script=sci_arttext\&pid $=$ S0103-84782008000300015 $>$. Acesso em: 13 jun. 2014. doi: $10.1590 / \mathrm{S} 0103-84782008000300015$.

MINUZZI, R.B.; LOPEZ, R.Z. Variabilidade de índices de chuva nos estados de Santa Catarina e Rio Grande do Sul. Bioscience Journal, v.30, p.697-706, 2014.

SIEGEL, S.; CASTELLAN JÚNIOR, N.J. Estatística nãoparamétrica para ciências do comportamento. 2.ed. Porto Alegre: Artmed, 2006. 448p.

STEEL, R.G.D. et al. Principles and procedures of statistics a biometrical approach. 3.ed. Nova York: McGraw-Hill, 1997. 666p.

THOM, H.C.S. Some methods of climatological analysis. Genève: World Meteorological Organization, 1966. 54p.

VERNICH, L.; ZUANNI, F. About the minimum number of years required to stabilize the solar irradiation statistical estimates. Solar Energy, v.57, p.445-447, 1996. Disponível em: <http:// dx.doi.org/10.1016/S0038-092X(96)00121-1>. Acesso em: 14 jun. 2014. doi: 10.1016/S0038-092X(96)00121-1. 